\title{
Circuit
}

Musiques contemporaines

\section{Silences, silence}

\section{Gilles Tremblay}

Volume 6, numéro 1, 1995

Tremblay/Varèse/Messiaen : Gilles Tremblay analyste

URI : https://id.erudit.org/iderudit/902116ar

DOI : https://doi.org/10.7202/902116ar

Aller au sommaire du numéro

Éditeur(s)

Les Presses de l'Université de Montréal

ISSN

1183-1693 (imprimé)

1488-9692 (numérique)

Découvrir la revue

Citer cet article

Tremblay, G. (1995). Silences, silence. Circuit, 6(1), 9-14.

https://doi.org/10.7202/902116ar

\section{Résumé de l'article}

La responsable de ce numéro a rassemblé une collection de six textes d'analyse du compositeur québécois Gilles Tremblay. En annexe, on retrouve une bibliographie chronologique des textes et travaux consacrés à ce créateur.
Ce document est protégé par la loi sur le droit d'auteur. L’utilisation des services d’Érudit (y compris la reproduction) est assujettie à sa politique d'utilisation que vous pouvez consulter en ligne.

https://apropos.erudit.org/fr/usagers/politique-dutilisation/
Cet article est diffusé et préservé par Érudit.

Érudit est un consortium interuniversitaire sans but lucratif composé de l’Université de Montréal, l'Université Laval et l'Université du Québec à Montréal. Il a pour mission la promotion et la valorisation de la recherche. https://www.erudit.org/fr/ 


\section{Gilles Tremblay : analyses}

\section{Silences, silence}

Conférence présentée à l'université Laval, à Québec, le 9 avril 1994 dans le cadre du Colloque sur l'écologie sonore: De l'expressivité du silence à la pollution par la musique. Inédit.

J'esquisserai ici quelques voies d'exploration sur les silences musicaux, puis de façon plus générale sur le caractère des silences (positifs et négatifs). Enfin, j'évoquerai un silence en particulier, car il est singulièrement éclairant pour notre réflexion, celui du récit $d$ ' « Élie au mont Horeb».

Multiplicité du silence.

En musique comme dans la vie, il y en a trois sortes principales: avant, pendant et après les événements.

\section{Avant}

- Le silence-anacrouse, comme au début de la Cinquième symphonie de Beethoven :

Exemple 1

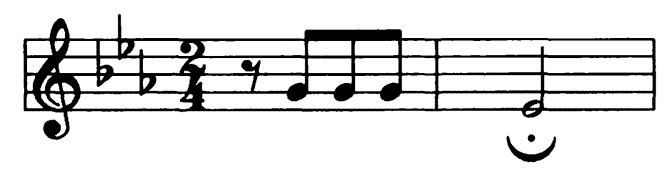

- ou pour préparer un nouveau thème : 
Exemple 2
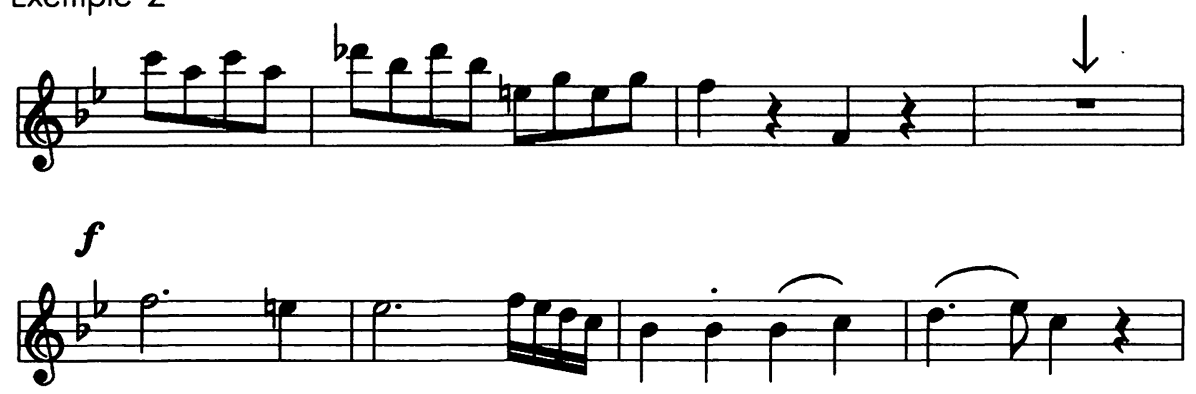

Mozart, Symphonie en sol mineur, n 40, K. 550, «Allegro», mesures 40-47.

- le silence qui précède, à la naissance, le premier cri du nouveau-né, donc le silence millénaire de cette personne-là, c'està-dire de nous tous.

\section{Pendant}

- Comme à la fin de l'« Adagio » du Concerto K. 488 de Mozart, où la musique est comme entourée d'une aura immense de silence, mise en relief par la ténuité et la brièveté même des pizzicati (mesures 84 et suivantes). On trouve aussi ce « sertissage » de silence dans une grande partie de l'œuvre de Webern "II:

- par la présence silencieuse d'un thème dans son absence. La mélodie s'entend par réflexe de l'auditeur mais elle est silencieuse. Exemple : le contrechant de l's Allegretto " de la Septième symphonie de Beethoven, à la toute fin sur restes en lambeaux du thème principal. Comme le chien de Pavlov, on salive la mélodie par association d'idées...!

Exemple 3

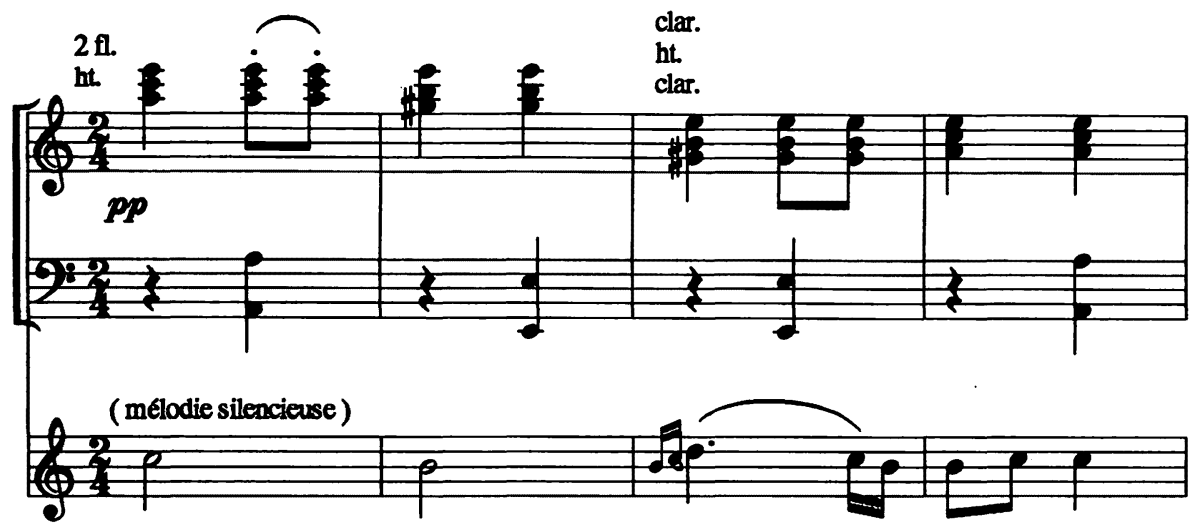

Beethoven, Septième symphonie, "Allegretto ", mesures 255 et suivantes. 
- par des silences en creux, comme dans Boulez, où les silences sont traités en négatif des sons (Deuxième sonate, pour piano), ou dans Machaut dans ses multiples traitements des hoquets (Messe de Notre-Dame, Hoquetus David);

- par des mélodies par manques : les silences creusant successivement les sons d'un bloc sonore, comme dans Messiaen;

Exemple 4

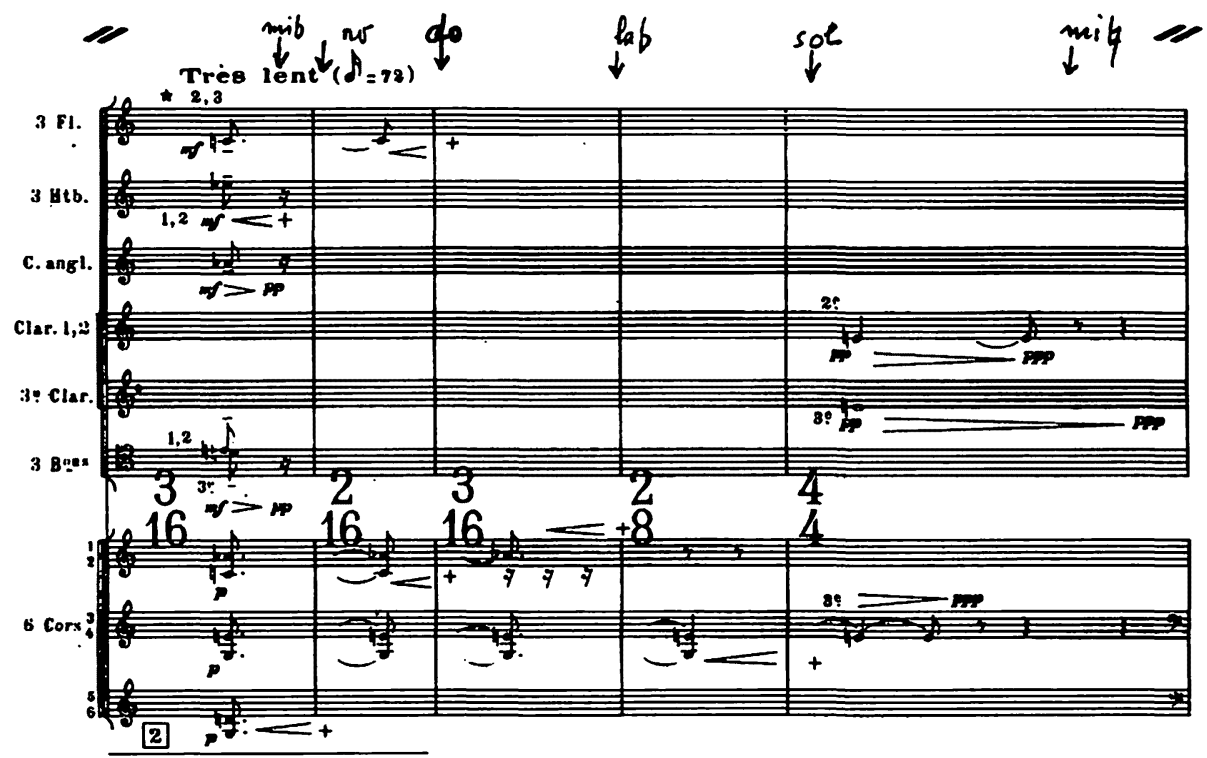

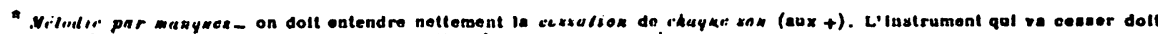
felre on loger erescondo, presque au momeat d'arróter, at sana axegeratlon.

A.l. 22.681

- par le silence qui se trouve entre deux événements liés entre eux, comme l'éclair (événement visuel) et le tonnerre (événement sonore). C'est un silence trait d'union.

\section{Après}

Depuis le naturel d'un diminuendo où le pianissimo est relayé par le silence, jusqu'au cri, où l'événement-choc crée un silence par stupéfaction; ou bien l'arrêt subit d'une musique au moment où l'on s'attend à un accent, comme le silence abrupt qui suit l'énorme échafaudage polyrythmique du "Cortège du sage », qui vous coupe presque le souffle /dans Stravinski, Le Sacre du Printemps, au chiffre 711 .

L'\& instant d'après», celui qui arrive après une catastrophe: c'est le titre d'une œuvre de Xavier Darasse (organiste et compositeur), à la suite d'un 
terrible accident. Sur un autre registre, certains silences-surprises sont particulièrement éloquents à cet égard. Pensons à celui qui a suivi le « Vive le Québec libre! » du général de Gaulle, entre la fin de la phrase et les applaudissements!

Si l'on passe à un plan plus général, et macroscopique, on pense à la célèbre phrase de Pascal: "Le silence éternel de ces espaces infinis m'effraie », sorte d'agoraphobie du silence.

II y a aussi le silence lourd: " un silence de mort», et le silence imposé : "Silence dans la salle !»

"La loi du silence ", l'omerta de la mafia, associée à la terreur.

Ce type de silence-terreur et son contraire, le bruit assourdissant, ont en commun un paradoxe : ils interdisent toute communication. $A$ la limite, ils sont mortels, dans la mesure où l'on ne peut vivre sans communiquer.

Nous lui opposerons le silence convivial. En général, il est coloré, vivant et peuplé. Ce n'est pas un silence pur, total (et totalitaire), mais plutôt un silence vibrant, habité, un silence de plénitude : « Regard du silence. Silence dans la main, arc-en-ciel renversé... chaque silence de la crèche révèle musique et couleurs qui sont les mystères de Jésus-Christ... » (Vingt regards sur l'Enfant-Jésus d'Olivier Messiaen.) Ce silence s'entend. II s'écoute. II n'est pas rien. Même la pièce de John Cage qui s'intitule Silence s'exécute au piano... On a donc le silence du piano, moment où l'on entend tout ce qui se passe autour.

Silences avant, pendant, après, silences imposés ou silences conviviaux, on pourrait énumérer toutes sortes de silences en les classant par catégories, espèces et sous-espèces afin d'en établir le répertoire: un traité du silence reste à faire (ce serait un projet à la fois passionnant et d'une grande nécessité). Cependant, à travers les silences esquissés, qui sont d'une autre nature qu'une simple absence du son, se dégagent déjà deux tendances : silences positifs, silences négatifs, qui peuvent nourrir notre réflexion. Pour mieux l'aiguiller, i'aimerais vous convier à un silence tout particulier. II m'a guidé dans la composition d'Avec, wampum symphonique (pour solistes, chœur, et grand orchestre), et il la conclut. Les quarante-cinq minutes qui le précèdent en sont, d'une certaine manière, la préparation convergente. Le mot même de «silence » et le silence qui suit en sont l'aboutissement (cf. exemple 5). II se trouve dans un des textes dont je me suis servi, tissé à travers ceux des fondateurs et des wampums amérindiens, et sert d'assise aux quatre moments principaux. C'est le récit d' «Élie au mont Horeb " (un rêveur lui aussi, comme les fondateurs et les amérindiens) tiré du Premier Livre des Rois $(19,11-13)$ :

Il lui fut dit:

Sors dans la montagne et tiens-toi devant Yahvé, car il va passer. 
... il y eut un ouragan, si fort et si violent qu'il fendait les montagnes et brisait les rochers.

... Mais... Yahvé n'était pas dans l'ouragan.

...et après l'ouragan il y eut :

un tremblement de terre

... mais...Yahvé n'était pas dans le tremblement de terre.

... et après le tremblement de terre : un feu

... mais... Yahvé n'était pas dans le feu.

Il se fit

alors

un merveilleux silence.

Ce silence n'est pas un silence vide. On le traduit parfois par « le bruit d'une brise légère $»$, comme dans la Bible de Jérusalem. Mais littéralement, il s'agit d'un silence vivant, d'une douceur infinie, situé ailleurs, sur un autre plan que celui des puissances de l'ouragan, du tremblement de terre et du feu. Inutile de dire que la chose la plus difficile à écrire a paradoxalement été de composer ce silence, donc de le préparer.

Ce merveilleux silence prend diverses formes dans l'espace et dans le temps. Dans l'espace des villes, ce sont des cours intérieures, des aires protégées des bruits, des lieux de contemplation aux vastes volumes architecturaux que sont les églises, synagogues ou mosquées; à la campagne : la forêt profonde, les sources d'eau, les vallées retirées. Espaces nourriciers. Dans le temps, ce sont les divers moments de pause de la journée et celui, privilégié, de la nuit, ainsi que certaines périodes de l'année ou de la vie.

Aux antipodes des musiques d'ameublement que Satie a si bien let si tragiquement) caricaturées, véritable conditionnement sonore qui est une atteinte à la liberté, ce silence-là appartient aux valeurs qu'une société doit cultiver. On peut donc parler ici, dans une perspective équilibrée d'écologie sonore, d'une véritable culture du silence.

Tel m'apparaît le sens de ce colloque. 
Exemple 5

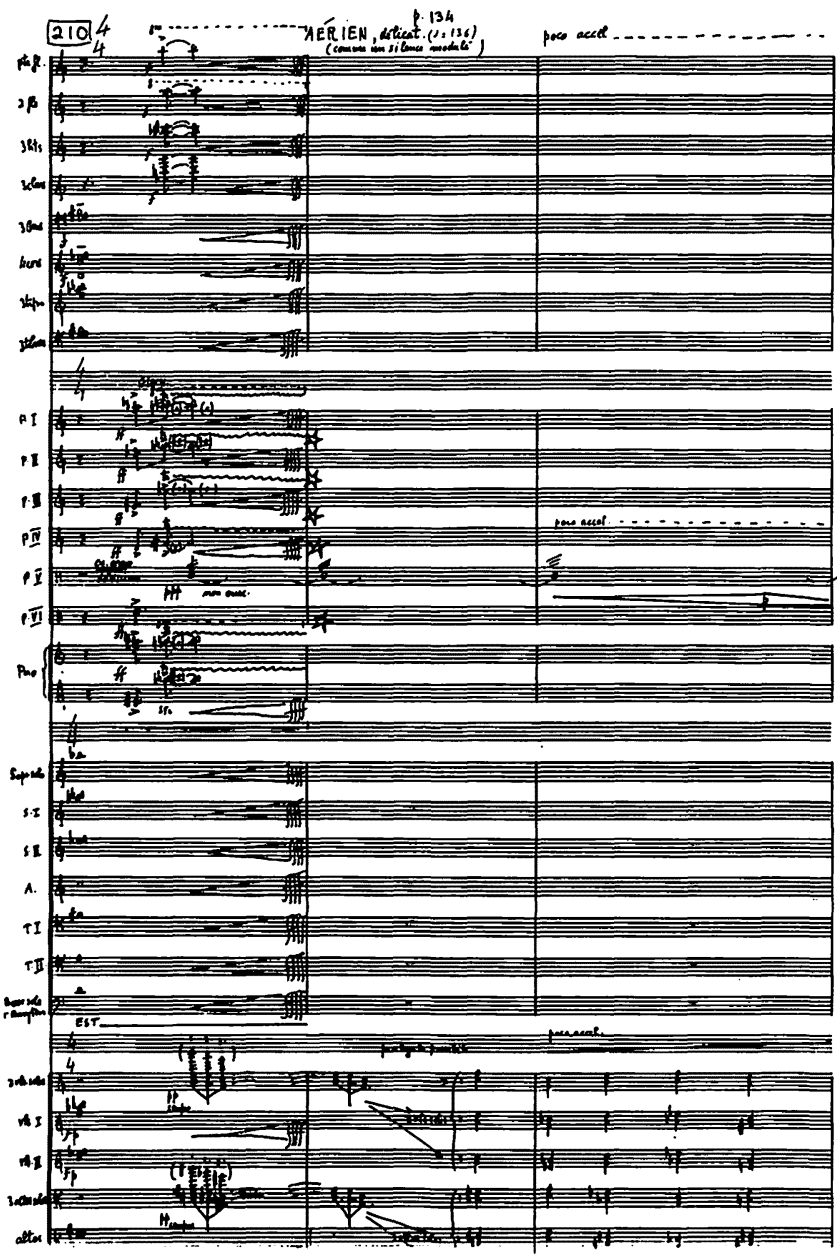

p. 135

soconith

$(-\cdots$

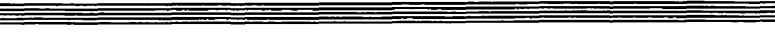
(1)

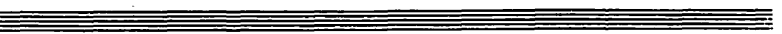
(2) ele (12)

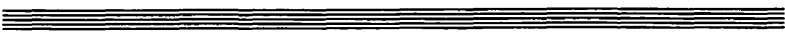

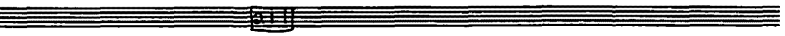

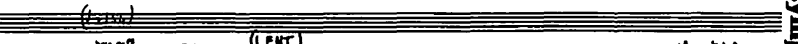

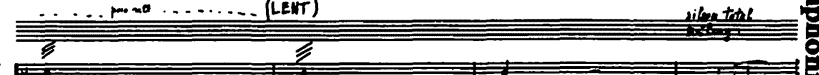

P.卫 (2)

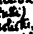

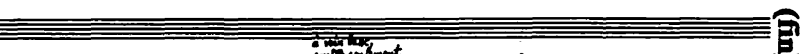
Pr isation.

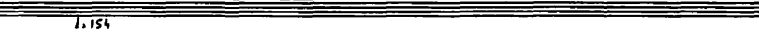
2 inin ${ }^{2}$

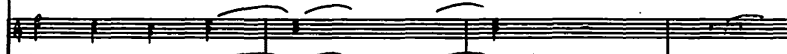
1 (1)

, Gillentrentlay $+1$

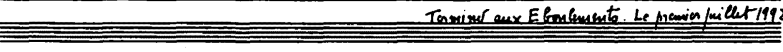
ele  